http://dx.doi.org/10.4314/ajtcam.v10i2.21

\title{
A RANDOMIZED, DOUBLE-BLIND, PLACEBO-CONTROLLED TRIAL OF A CHINESE HERBAL SOPHORA FLOWER FORMULA IN PATIENTS WITH SYMPTOMATIC HAEMORRHOIDS: A PRELIMINARY STUDY
}

\author{
Kee-Ming Man, ${ }^{1,2,3}$ Wen-Chi Chen, ${ }^{4,5 *}$ Hwei-Ming Wang, ${ }^{6}$ Huey-Yi Chen, ${ }^{4,5}$ Jui-Lung Shen, ${ }^{6}$ \\ Lieh-Der Chen, ${ }^{4}$ Fuu-Jen Tsai, ${ }^{4,5}$ Yung-Hsiang Chen, ${ }^{4,5}$ De-Xin Yu, ${ }^{2, *}$ and Feng-Fan Chiang ${ }^{4,6,7, *}$
}

\author{
${ }^{1}$ Department of Anesthesiology, Tungs' Taichung MetroHarbor Hospital, Taichung 43503, Taiwan, \\ ${ }^{2}$ Graduate Institute of Geriatric Medicine, Department of Urology, First Affiliated Hospital, Anhui \\ Medical University, Hefei 230022, China \\ ${ }^{3}$ Department of Life Sciences, National Chung Hsing University, Taichung 40227, Taiwan, ${ }^{4}$ Graduate \\ Institute of Integrated Medicine, Department of Post Baccalaureate Chinese Medicine, School of \\ Chinese Medicine, College of Chinese Medicine, China Medical University, Taichung 40402, Taiwan, \\ ${ }^{5}$ Departments of Urology, Obstetrics and Gynecology, Medical Genetics and Pediatrics, and Medical \\ Research, China Medical University Hospital, Taichung 40402, Taiwan, ${ }^{6}$ Division of Colorectal \\ Surgery, Department of Surgery, Department of Dermatology, Taichung Veterans General Hospital, \\ Taichung 40705, Taiwan, ${ }^{7}$ School of Nutrition, Chung Shan Medical University, Taichung 40201, \\ Taiwan \\ *E-mail: hankel.chiang@gmail.com and yhchen@mail.cmu.edu.tw
}

\begin{abstract}
Dried flowers and buds of Sophora japonica (Huaihua) are used in China, Japan and Korea for treating haematemesis and bleeding haemorrhoids. This study compared the clinical safety and efficacy of a Sophora flower formula with a placebo for the conservative treatment of symptomatic haemorrhoids. The study was a prospective, double-blind, randomized placebo-controlled trial. The clinical effective rate, symptom score and the incidence of important clinical events were used as observation indices to evaluate the effect of the Sophora flower formula. The results showed that after 7 days of treatment, improvement was observed in $\mathbf{8 7 . 0 \%}$ of the patients' major symptoms in the Sophora flower formula group compared with $81.8 \%$ of those in the placebo group. After 14 days, $78.2 \%$ patients in the Sophora flower formula group were asymptomatic, whereas $40.9 \%$ of those in the placebo group exhibited residual symptoms. However, the difference between both groups was not statistically significant. As the bowel habits of the patients improved and as the patients took sitz baths, their symptoms improved drastically, regardless of the use of the Sophora flower formula. These findings indicate that the traditional Chinese Sophora flower formula is clinically safe; however, its effects on haemorrhoids need to be studied in a larger sample size and with different dosages. The present study results may be a potential clinical reference for physicians prescribing medications for patients with symptomatic haemorrhoids.
\end{abstract}

Keywords: Chinese herbal medicine, clinical trial, hemorrhoids, Sophora flower (Sophora japonica)

\section{Introduction}

Haemorrhoids are a common condition and are defined as vascular structures that extend from the subcutaneous arteriovenous vascular plexus (Lao et al., 2005; Loder et al., 1994; Thulesius and Gjores, 1973) in the anal region to the smooth muscle of the anal sphincter through the conjoined longitudinal muscle (Hansen, 1976; Thomson, 1975). They generate $15 \%-20 \%$ anal resting pressure and contribute to the complete closure of the anus (Lestar et al., 1989). Several studies have revealed that patients with haemorrhoids tend to have a high anal resting pressure (Loder et al., 1994; Read et al., 1982; Sun et al., 1990). Symptoms of haemorrhoids include pain, haemorrhage and prolapse and itchiness of the anus (Loder et al., 1994), which are usually caused by swollen haemorrhoidal tissues or a swollen vascular plexus, both of which are susceptible to injury.

Many studies have discussed the surgical treatment of haemorrhoids but very few have focussed on less invasive and safer medical treatments such as increasing dietary fibre intake, preventing constipation and taking sitz baths (Lorenzo-Rivero, 2009). Evidence from clinical trials indicates that diets containing fibres such as psyllium can soften the stool and reduce pain and haemorrhaging. However, the efficacy of such treatments remains to be proven because diarrhoea can occasionally induce the onset of haemorrhoids (Broader et al., 1974; Perez-Miranda et al., 1996; Webster et al., 1978). Therefore, there is scope for improvement in the field of medical treatment of haemorrhoids (Tchacondo et al., 2011).

Dried flowers and buds of Sophora japonica (Huaihua) are used in China, Japan and Korea for treating haematemesis and bleeding haemorrhoids. The traditional Chinese Sophora flower formula comprises 4 kinds of herbs-Sophorae flos, Cacumen platycladi, Schizonepeta tenuifolia and Citrus aurantium. These herbs can be used for treating haemorrhoids (Chen and Hsieh, 2010; Ishida et al., 1989) and colitis (Tong et al., 2010; Zhao et al., 2011). Studies have shown that S. flos, C. platycladi and S. tenuifolia can considerably reduce the haemorrhaging and coagulation time and effectively stop haemorrhaging (Al-Suhaimi et al., 2011). C. aurantii can enhance gastrointestinal contraction rhythm and prevent 
http://dx.doi.org/10.4314/ajtcam.v10i2.21

constipation (Fang et al., 2009; Huang et al., 2010). Therefore, the combination of these herbs in the Sophora flower formula has a remarkable effect on haemorrhoids. However, no standardized research on the application of this formula in haemorrhoid treatment has been conducted. Therefore, the present study adopted a double-blind, randomized placebo-controlled approach to assess the clinical efficacy and safety of the Sophora flower formula for treating symptomatic haemorrhoids compared with the conventional conservative treatment. The results of this clinical trial may be a potential reference material for physicians treating patients with symptomatic haemorrhoids.

\section{Methods \\ Patients}

The study was conducted from February to May 2008 at the outpatient unit of the Department of Colorectal Surgery at Taichung Veterans General Hospital, Taichung, Taiwan. The trial protocol (No. C07072) was reviewed and approved by the institutional review board (IRB). A total of 48 patients with symptomatic haemorrhoids were included in the study; a written informed consent was obtained from the patients. A double-blind, randomized placebo-controlled design was adopted to investigate the clinical efficacy and safety of the conservative medical treatment of symptomatic haemorrhoids with and without the use of the Sophora flower formula. All patients were above 18 years of age, were diagnosed with haemorrhoids and had received and complied with the conservative medical treatment. The exclusion criteria were as follows: $4^{\text {th }}$-degree haemorrhoids; pregnancy; apparent coagulation abnormalities or kidney impairment accompanied with colorectal diseases such as cancer, Crohn's disease, ulcerative colitis, anal fistula, anal fissure, proctitis or perineal skin inflammation; previous pelvic electrotherapy or non-conservative treatment within the previous 6 months (rubber band ligation, infrared therapy and freeze therapy); medication use for less than half the treatment duration.

The patients in both groups underwent an information-based health education session regarding diet and lifestyle. Those with irregular bowel habits were treated with Sophora flower formula or placebo and both groups were instructed to take a 10-min sitz bath 4 times a day (recommended times were after waking, at 1:00 pm, 7:00 pm and before sleeping).

\section{Sample Size Determination}

We estimated the sample size required to detect a $20 \%$ difference in the improvement of the major symptom using data from a previous systematic review and meta-analysis study. (Chen and You, 2010). We calculated that we would require a sample size of 22 patients in each group to achieve $80 \%$ power at $\alpha=0.05$. Anticipating a drop-out rate of $10 \%$, we accordingly adjusted the sample size to 24 patients for each group.

\section{Medication}

The Sophora flower formula (concentrated scientific Chinese herbal medicine) comprises a combination of S. flos, C. platycladi, S. tenuifolia Briq. and C. aurantium L. in the ratio 1:1:1:1. All the water soluble herbal ingredients were used in equal amounts, were ground into a fine powder and vacuum dried. The Sophora flower formula and placebo capsules were similar in appearance and were manufactured by Sun Ten Pharmaceutical Co. Ltd., Taichung, Taiwan, approved by Taiwan government's Department of Health. The Sophora flower formula was qualified by fingerprint analysis using high-performance liquid chromatography (Figure 1). The pharmaceutical company analysed the standard component markers to determine whether each Sophora flower formula had the same components for quality control (data not shown). A 2-g dose of the Sophora flower formula or placebo was administered 3 times a day (in the morning, at noon and in the evening) for 2 weeks.

The capsules were enclosed within a sealed packages that indicated the name of the pharmaceutical company, clinical trial number, principal investigator's name, patient number, drug quantity, instructions for use, expiry date, batch number and storage conditions; they were labelled 'for clinical trial use only'. The shelf-life of the test drugs was printed on the packages to ensure that they were taken within the effective period. The test drug was stored in a sealed container and placed in a dry, cool location at a temperature between $15^{\circ} \mathrm{C}$ and $30^{\circ} \mathrm{C}$. Patients were randomly assigned to 2 test groups by permuted-block randomization using a computer program. On assignment, 1 capsule was removed from each drug bottle for follow-up purposes.

\section{Assessments}

All participants and researchers, including the investigator, were blinded to the study randomization procedure. A well-trained investigator evaluated the haemorrhoids and performed the anoscopy in both patient groups. The primary outcome was measured as the improvement in signs and symptoms of the syndrome, such as bleeding scores, swelling and itching. Secondary outcome were measured as the rate of and time to recurrence. The incidence of haemorrhage, pain, itchiness and a swelling sensation was recorded before, during and after the treatment. The assessment time points were post-treatment days 7, 14, 28 and 56. The assignment scores were as follows: 2 for major symptoms and 3 for other symptoms; 2 for worsened symptoms; 1 for persistent and unchanged symptoms; 0 for improved, mild residual symptoms or no symptoms. All symptoms were recorded and assessed by the same investigator. The patients underwent an anoscopy on their $1^{\text {st }}$ visit and their return visit after completion of the treatment program; all findings were recorded. If symptom(s) persisted for more than 14 days, the patient received an alternative treatment. If anoscopy revealed inflammation in the anal canal, the treatment was considered to have failed and the symptoms to have persisted. The assessment of adverse clinical events included the following: (1) monitoring vital signs and clinical indicators for major changes (2) analysing changes in laboratory data and (3) recording symptomatic adverse reactions including gastrointestinal symptoms such as upper 
http://dx.doi.org/10.4314/ajtcam.v10i2.21

abdominal pain and a burning sensation in the stomach; psychological symptoms such as headache, lethargy and tinnitus; circulatory symptoms such as palpitations; respiratory symptoms such as asthma; urinary symptoms such as oliguria and haematuria and skin rash and oedema. All adverse events were recorded in detail. The principal investigator informed the IRB within 24 hours of any serious adverse events that could cause death or a life-threatening or permanent injury, warranted admission or extended hospitalization, were capable of inducing congenital malformations and other types of malignancy, or were caused by overdose. In the case of non-serious adverse events not listed above, the principal investigator filled an Adverse Event Form and reported the event to the IRB as an isolated case.

\section{Patient Withdrawal}

The patients were free to withdraw from the trial at any time. Withdrawal did not affect the doctor-patient relationship or jeopardize any medical rights of the patient. The patient could request for necessary follow-up examinations mandatory for the part of the trial that he/she participated in, even after withdrawal from the trial.

\section{Trial Procedure}

At the time of enrolment, the eligible patients underwent a complete basic examination and symptom assessment, attended an information-based health education session and signed the informed consent form. They returned any remaining medication and were re-assessed on post-treatment day 28. A telephone interview was conducted on post-treatment days 7 , 14 and 56. All the interviews were conducted and recorded by the same researcher. A flow chart of the trial procedure is shown in Figure 2.

\section{Statistical Analyses}

Continuous variables were used for descriptive statistical data such as the average and standard deviation. Categorical variables were used for data such as frequency and the corresponding ratio. All statistical tests were 2-sided, with the significance level set at 0.05 . The major population for the analysis of efficacy was the intent-to-treat population. Patient demographics and baseline values of continuous variables such as age and weight were compared between the patients in the Sophora flower formula and those in the placebo groups using the Student's $t$-test. Categorical variables such as gender were compared using the Chi-square test.

\section{Results \\ Comparison of General Efficacy}

A total of 45 patients with symptomatic haemorrhoids completed the clinical trial, 40 of these patients experienced the major symptom of anal haemorrhage. Other accompanying symptoms included anal pain (22 patients), itchiness (9 patients) and a swelling sensation (19 patients). The patients were randomly assigned to the Sophora flower formula group or placebo group by the Chinese and Western Clinical Trial Centre at our institution. The characteristics of the patients in both groups are listed in Table 1.

Table 1: Patient characteristics between Sophora flower formula and placebo groups

\begin{tabular}{|c|c|c|c|}
\hline & $\begin{array}{l}\text { Sophora flower formula group } \\
\qquad(n=23)\end{array}$ & $\begin{array}{l}\text { Placebo group } \\
\qquad(n=22)\end{array}$ & $p$ value \\
\hline Mean age (years \pm SD) & $37.3 \pm 12.7$ & $39.5 \pm 13.9$ & 0.595 \\
\hline Gender (male/female) & $18 / 5$ & $15 / 7$ & 0.669 \\
\hline Duration of disease (months \pm SD) & $39.5 \pm 45.1$ & $33.5 \pm 44.5$ & 0.667 \\
\hline \multicolumn{4}{|l|}{ Symptoms $[n(\%)]$} \\
\hline Rectal bleeding & $22(95.7 \%)$ & 18 (81.8\%) & 0.187 \\
\hline Anal pain & $13(56.5 \%)$ & $9(40.9 \%)$ & 0.454 \\
\hline Anal pruritus & $6(26.1 \%)$ & $3(13.6 \%)$ & 0.459 \\
\hline Tenesmus /mass feeling & $10(43.5 \%)$ & $9(40.9 \%)$ & 0.899 \\
\hline $\begin{array}{l}\text { Constipation or Irregular } \\
\text { bowel habit }\end{array}$ & $12(52.2 \%)$ & $12(54.5 \%)$ & 0.889 \\
\hline
\end{tabular}

The average age of patients in the treatment group was $37.3 \pm 12.7$ years. The group included 18 males and 5 females and their average disease duration was $39.5 \pm 45.1$ months. The major symptom of haemorrhage was reported by 22 patients, whereas pain, itchiness and a swelling sensation were reported by 13, 6 and 10 patients, respectively. Irregular bowel habits were reported by 12 patients. The average age of the patients in the placebo group was $39.5 \pm 13.9$ years. The group included 15 males and 7 females and their average disease duration was $33.5 \pm 44.5$ months. The major symptom of haemorrhage was reported by 18 patients, whereas pain, itchiness and a swelling sensation were reported by 9,3 and 9 patients, respectively. Irregular bowel habits were reported by 12 patients. Haemorrhoid symptoms were comparable in both groups because both were characterized by haemorrhaging. 
Man et al., Afr J Tradit Complement Altern Med. (2013) 10(2):343-351

http://dx.doi.org/10.4314/ajtcam.v10i2.21

Table 2. The main symptoms score in Sophora flower formula and placebo groups

\begin{tabular}{|c|c|c|c|c|}
\hline Time & Score & $\begin{array}{c}\text { Sophora flower formula group, } \\
n(\%)\end{array}$ & $\begin{array}{c}\text { Placebo group, } \\
n(\%)\end{array}$ & $p$ value \\
\hline Baseline & $\begin{array}{l}0 \\
1 \\
2 \\
3\end{array}$ & $\begin{array}{c}- \\
- \\
23(100 \%) \\
-\end{array}$ & $\begin{array}{c}- \\
- \\
22(100 \%) \\
-\end{array}$ & - \\
\hline 7 days & $\begin{array}{l}0 \\
1 \\
2 \\
3\end{array}$ & $\begin{array}{c}14(60.9 \%) \\
6(26.1 \%) \\
3(13.0 \%) \\
-\end{array}$ & $\begin{array}{c}12(54.5 \%) \\
6(27.3 \%) \\
4(18.2 \%) \\
-\end{array}$ & 0.872 \\
\hline 14 days & $\begin{array}{l}0 \\
1 \\
2 \\
3\end{array}$ & $\begin{array}{c}18(78.2 \%) \\
4(17.4 \%) \\
1(4.3 \%) \\
-\end{array}$ & $\begin{array}{c}13(59.1 \%) \\
5(22.7 \%) \\
3(13.6 \%) \\
1(4.5 \%)\end{array}$ & 0.408 \\
\hline 28 days & $\begin{array}{l}0 \\
1 \\
2 \\
3\end{array}$ & $\begin{array}{c}19(82.6 \%) \\
2(8.7 \%) \\
1(4.3 \%) \\
1(4.3 \%)\end{array}$ & $\begin{array}{c}14(63.6 \%) \\
3(13.6 \%) \\
3(13.6 \%) \\
2(9.1 \%)\end{array}$ & 0.518 \\
\hline 56 days & $\begin{array}{l}0 \\
1 \\
2 \\
3\end{array}$ & $\begin{array}{c}20(87.0 \%) \\
2(8.7 \%) \\
1(4.3 \%) \\
-\end{array}$ & $\begin{array}{c}17(77.3 \%) \\
1(4.5 \%) \\
2(9.1 \%) \\
2(9.1 \%)\end{array}$ & 0.409 \\
\hline
\end{tabular}

The major symptoms analysed in the comparison of both groups are shown in Table 2 . The baseline value was set at score \#2. After 7 days of treatment, the symptoms in $60.9 \%$ patients were alleviated and those in $26.1 \%$ patients improved in the Sophora flower formula group. In the placebo group, the symptoms in 54.5\% patients disappeared and those in $18.2 \%$ persisted without any improvement. After 14 days, 78.2\% patients in the Sophora flower formula group were asymptomatic, whereas $40.9 \%$ patients in the placebo group still exhibited residual symptoms. On day 28, symptom deterioration was observed in $9.1 \%$ patients in the placebo group. On day 56, $13.0 \%$ patients in the Sophora flower formula group suffered from a relapse of haemorrhoids. On an average, the major symptom disappeared after 3.1 days in the Sophora flower formula group and after 4.5 days in the placebo group.

Analysis and comparison of the total symptom scores revealed that the patients in the Sophora flower formula group had a tendency towards a lower rate of relapse than those in the placebo group and also showed an improvement in anal haemorrhage. However, there was no statistically significant difference between both groups.

\section{Adverse Reactions}

No significant abnormal changes were observed in biochemical tests such as routine blood and urine, kidney function and coagulation function tests performed before and after the treatment in both groups. One patient in the placebo group experienced mild kidney function impairment [creatinine, $1.7 \mathrm{mg} / \mathrm{dl}$ (normal range, $0.7-1.4 \mathrm{mg} / \mathrm{dl}$ )]. In addition, 1 patient in each group reported non-specific side effects including fatigue and dizziness 10 minutes after taking the medication; however, both these side effects disappeared rapidly. None of the patients experienced any significant change in blood pressure or heart rate. 
Man et al., Afr J Tradit Complement Altern Med. (2013) 10(2):343-351

http://dx.doi.org/10.4314/ajtcam.v10i2.21

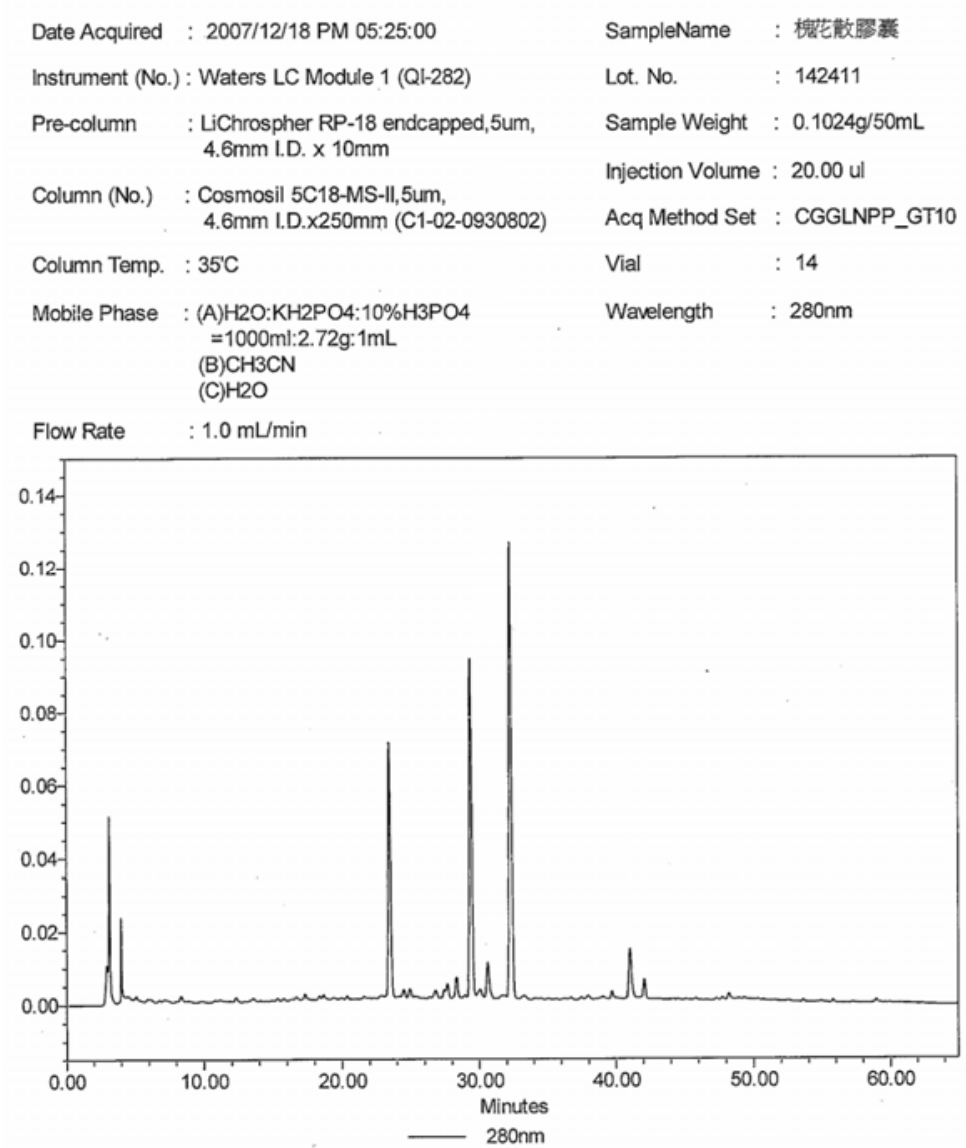

Figure 1: Typical high performance liquid chromatography chromatogram profile of extract from Sophora flower formula. Diode array detector set at absorbance UV $203 \mathrm{~nm}$.

\section{Patient Withdrawal}

A total of 3 patients withdrew from the trial. Two patients withdrew for unknown reasons during week 1 of the trial. One patient suffered from systemic allergic reactions including systemic urticaria, shortness of breath and dyspnoea on day 9 of the treatment. Upon emergency notification, IRB and the Chinese and Western Clinical Trial Centre at our institution disclosed that the patient was assigned to the placebo group. The patient was admitted to the Dermatology Ward and was discharged after 3 days when her condition stabilized. The diagnosis was non-specific urticaria induced by the seasonal environment. Since intermittent anal haemorrhaging persisted, the patient returned to the clinic at week 4 , and a rubber-band ligation surgery was indicated. This patient was excluded from the analysis because her randomization information was disclosed.

\section{Discussion}

Our results demonstrate that the traditional Chinese Sophora flower formula is clinically safe. However, the study failed to reveal any distinctive effect on symptoms such as pain, itchiness and swelling sensation. As the bowel habits of the patients improved and they took sitz baths, their symptoms improved drastically, regardless of the use of the Sophora flower formula. The effects of the Sophora flower formula on haemorrhoids should be further explored in a larger study population and with different dosages. The present study results could be a potential clinical reference for physicians treating patients with symptomatic haemorrhoids.

Haemorrhoids are common disorder, which do not have a definitive aetiology. The consensus is that the aetiology of haemorrhoids is multi-factorial and can be analysed from the point of view of the complex anal structure and function (Gan et al., 2010; Joshi and Neugebauer, 2010): The anus is the excretory outlet for faeces which are composed mainly of bacteria. It frequently sustains injuries such as rupture by hardened stool or abrasion by sanitary tissues. Therefore, a strong immune system is required to prevent bacterial invasion. Because immunity is supported by blood circulation, local unsanitary conditions or small lesions contribute to the worsening of the haemorrhoid condition. Therefore, a sitz bath ensures local cleanliness and decreases the local inflammatory response and vascular swelling, which ultimately produces a therapeutic effect. (Gervaz et al., 1995; Sobhani et al., 2002). S Furthermore, haemorrhoids exert major anal resting pressure causing the 


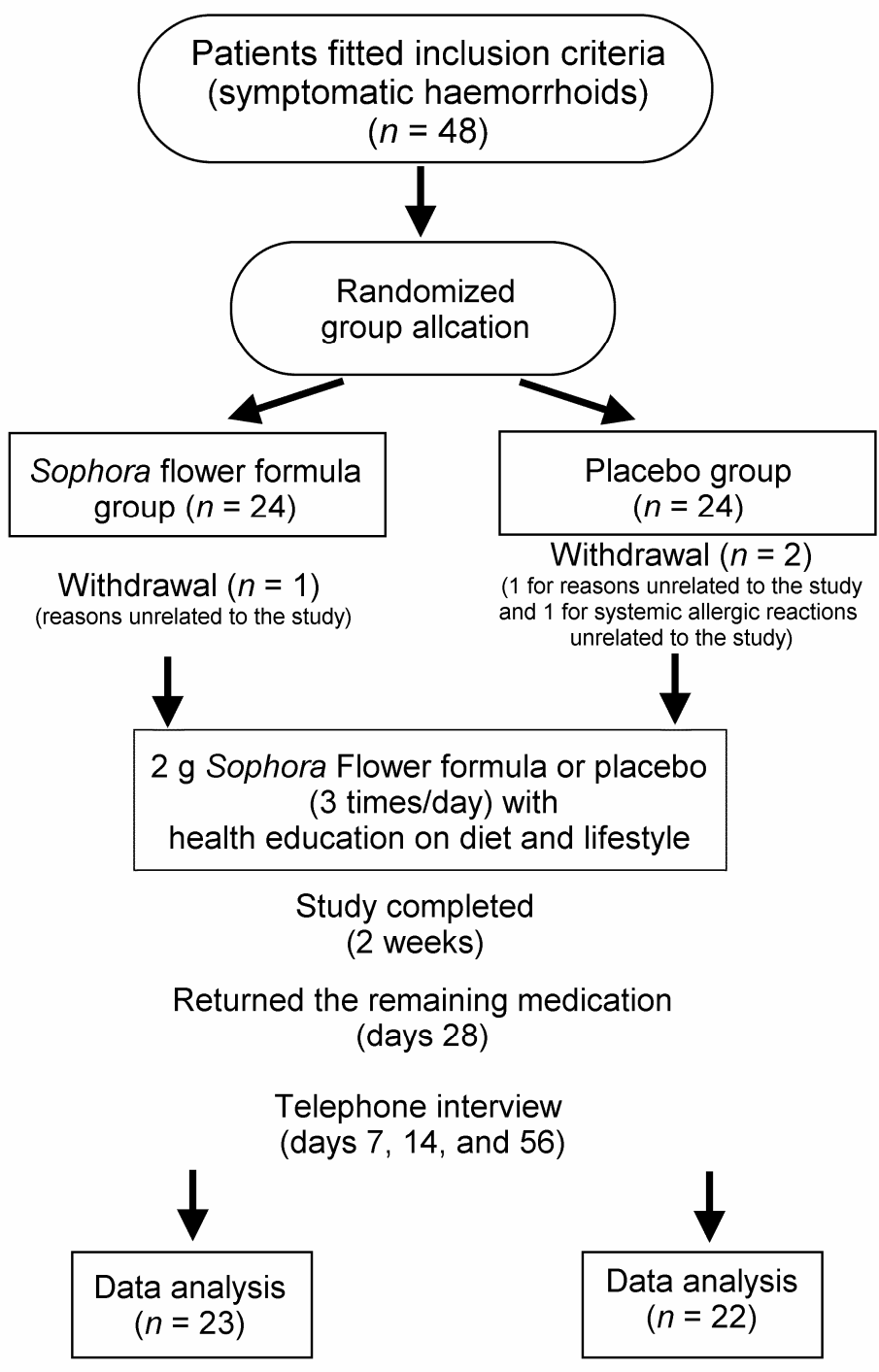

Figure 2: Flow chart of the trial.

closure of the anus. Therefore, constipation and increased abdominal pressure tend to worsen the haemorrhoids, causing their enlargement. In other words, enhancing the body's immune system can help reduce the excessive congestion of local haemorrhoids, thus facilitating an early recovery (Anderson et al., 2009; Holzheimer, 2004).

Traditional Chinese medicines such as the Sophora flower formula can improve the physical state and immune system of the patient. The Sophora flower formula can therefore exert a certain therapeutic effect on haemorrhoids (Chen and Hsieh, 2010). However, limited clinical research has been conducted on the therapeutic effect of this formula. Therefore, the present study was designed to verify the therapeutic effect of the Sophora flower formula on haemorrhoids using a scientific approach. The patients randomized to the Sophora flower formula and the placebo groups were young and did not suffer from severe structural changes typically observed in $3^{\text {rd }}$ - or $4^{\text {th }}$-degree haemorrhoids. Enlarged and prolapsed tissues are prone to injury during excretion of hardened stool, both of which may lead to swelling and bleeding of the tissues and even result in the formation of thrombotic haemorrhoids. The clinical consensus is that patients with such injuries should be treated with a more aggressive approach such as surgery, rubber band ligation or injection of stool softeners to reduce hyperplasia or restore the prolapsed tissues due to local responses (Altomare et al., 2006).

The study population included a higher number of male patients than female patients; most males were characterized by the acute onset of haemorrhoids. Males have well-developed perineal pelvic muscles and those with greater anal strip muscle tone show distinct symptoms even with mild swelling of haemorrhoidal tissues. Improvement was especially evident for the symptom of haemorrhage and no remarkable side effects were observed. The mechanism may be related to the regulation of cytokines in the systemic and haemorrhoidal region, which increases the permeability of the blood vessels and improves the coagulation mechanism and lymphatic blood pressure (Haas et al., 1984; Szlavy et al., 1990). Current medical treatments for haemorrhoids in the literature include an oral micronized purified flavonoid fraction (Buckshee et al., 1997; Cospite, 1994; Ho et al., 1995; Mentes et al., 2001; Meyer, 1994; Misra and Parshad, 2000) and dobesilate (Haas et al., 1984; Szlavy et al., 
http://dx.doi.org/10.4314/ajtcam.v10i2.21

1990).

Haemorrhoids are a benign and self-limiting condition, and clinically, the symptoms usually disappear rapidly and show few relapses. Treatments with the least number of side effects are the best option. Oral medications combined with local treatments and modifications in daily diet are the most accepted options (La Torre and Nicolai, 2004). The conservative treatment of haemorrhoidal haemorrhaging recommended by current evidence-based medicine is an increase in the intake of fibre to prevent constipation (Misra and Imlitemsu, 2005) and sitz baths to ease anal discomfort and itchiness (Alonso-Coello et al., 2005). With regard to oral medications, we propose an alternative to Daflon and dobesilate. Preliminary evidence confirmed that the Sophora flower formula can help alleviate symptoms and reduce the relapse tendency when used for treating symptomatic haemorrhoids. Such evidence was supported by a double-blind, randomized placebo-controlled pilot study that comprised both subjective patient perception and objective anoscopy.

Some limitations of this study should be noted. First, the sample size was small and the experimental design was too simple without further analysis, which affects the reliability of this study. Second, only a short treatment period for symptomatic haemorrhoids was investigated. Further studies are required to verify the efficacy and safety of the formula for symptom management on a larger scale, for a longer duration, with different dosages and after surgery in different races.

\section{Conclusion}

The traditional Chinese herbal Sophora flower formula is clinically safe. However, as the bowel habits of the patients improved and they took sitz baths, their symptoms improved drastically regardless of the use of the Sophora flower formula. Therefore, the study failed to demonstrate a distinctive effect of this formula on symptoms such as pain, itchiness and swelling sensation. However, study results could serve as a potential clinical reference for physicians treating patients with symptomatic haemorrhoids.

\section{Financial Support}

This study was supported in part by a grant CMU99-COL-01-2 from the China Medical University Hospital, grants NSC 98-2314-B-039-023-MY3 and NSC 100-2320-B-039-008-MY2 from the National Science Council, and grant DOH101-TD-B-111-004 from the Taiwan Department of Health Clinical Trial and Research Center of Excellence. We thank Miss Jin-Mei Wang and Chi-Hsiang Wei for experimental assistance.

\section{References}

1. Al-Suhaimi E.A., Al-Riziza N.A., Al-Essa R.A. Physiological and therapeutical roles of ginger and turmeric on endocrine functions. Am. J. Chin. Med. 39: 215-31. 2011.

2. Alonso-Coello P., Guyatt G., Heels-Ansdell D., Johanson J.F., Lopez-Yarto M., Mills E., Zhou Q. Laxatives for the treatment of hemorrhoids. Cochrane Database Syst. Rev.: CD004649. 2005.

3. $\quad$ Altomare D.F., Rinaldi M., La Torre F., Scardigno D., Roveran A., Canuti S., Morea G., Spazzafumo L. Red hot chili pepper and hemorrhoids: the explosion of a myth: results of a prospective, randomized, placebo-controlled, crossover trial. Dis. Colon Rectum 49: 1018-23. 2006.

4. Anderson J.W., Baird P., Davis R.H., Jr., Ferreri S., Knudtson M., Koraym A., Waters V., Williams C.L. Health benefits of dietary fiber. Nutr. Rev. 67: 188-205. 2009.

5. Broader J.H., Gunn I.F., Alexander-Williams J. Evaluation of a bulk-forming evacuant in the management of haemorrhoids. The British journal of surgery 61: 142-4. 1974.

6. $\quad$ Buckshee K., Takkar D., Aggarwal N. Micronized flavonoid therapy in internal hemorrhoids of pregnancy. Int. J. Gynaecol. Obstet. 57: 145-51. 1997.

7. Chen H.N., Hsieh C.L. Effects of Sophora japonica flowers (Huaihua) on cerebral infarction. Chin. Med. 5: 34. 2010.

8. Chen J.S., You J.F. Current status of surgical treatment for hemorrhoids--systematic review and meta-analysis. Chang Gung Med. J. 33: 488-500. 2010.

9. Cospite M. Double-blind, placebo-controlled evaluation of clinical activity and safety of Daflon $500 \mathrm{mg}$ in the treatment of acute hemorrhoids. Angiology 45: 566-73. 1994.

10. Fang Y.S., Shan D.M., Liu J.W., Xu W., Li C.L., Wu H.Z., Ji G. Effect of constituents from Fructus Aurantii Immaturus and Radix Paeoniae Alba on gastrointestinal movement. Planta Med. 75: 24-31. 2009. 
http://dx.doi.org/10.4314/ajtcam.v10i2.21

11. Gan T., Liu Y.D., Wang Y., Yang J. Traditional Chinese Medicine herbs for stopping bleeding from haemorrhoids. Cochrane Database Syst. Rev.: CD006791. 2010.

12. Gervaz E., Dauge-Geffroy M.D., Sobhani I., Vissuzaine C., Mignon M., Benhamou G., Potet F. Quantitative analysis of the immune cells in the anal mucosa. Pathol. Res. Pract. 191: 1067-71. 1995.

13. Haas P.A., Fox T.A., Jr., Haas G.P. The pathogenesis of hemorrhoids. Dis. Colon Rectum 27: 442-50. 1984.

14. Hansen H.H. [The importance of the Musculus canalis ani for continence and anorectal diseases (author's transl)]. Langenbecks Archiv fur Chirurgie 341: 23-37. 1976.

15. Ho Y.H., Foo C.L., Seow-Choen F., Goh H.S. Prospective randomized controlled trial of a micronized flavonidic fraction to reduce bleeding after haemorrhoidectomy. Br. J. Surg. 82: 1034-5. 1995.

16. Holzheimer R.G. Hemorrhoidectomy: indications and risks. Eur. J. Med. Res. 9: 18-36. 2004.

17. Huang W., Huang X., Xing Z., Qiu X., Wang Y., Fan R., Liu W., Ren P., Liu Z., Zhou H. Meranzin Hydrate Induces Similar Effect to Fructus Aurantii on Intestinal Motility through Activation of H(1) Histamine Receptors. J. Gastrointest. Surg. 2010.

18. Ishida H., Umino T., Tsuji K., Kosuge T. Studies on the antihemostatic substances in herbs classified as hemostatics in traditional Chinese medicine. I. On the antihemostatic principles in Sophora japonica L. Chem. Pharm. Bull. (Tokyo) 37: 1616-8. 1989.

19. Joshi G.P., Neugebauer E.A. Evidence-based management of pain after haemorrhoidectomy surgery. Br. J. Surg. 97: 1155-68. 2010.

20. La Torre F., Nicolai A.P. Clinical use of micronized purified flavonoid fraction for treatment of symptoms after hemorrhoidectomy: results of a randomized, controlled, clinical trial. Dis. Colon Rectum 47: 704-10. 2004.

21. Lao C.J., Lin J.G., Kuo J.S., Chao P.D., Cheng C.Y., Tang N.Y., Hsieh C.L. Microglia, apoptosis and interleukin-1beta expression in the effect of sophora japonica l. on cerebral infarct induced by ischemia-reperfusion in rats. Am. J. Chin. Med. 33: 425-38. 2005.

22. Lestar B., Penninckx F., Kerremans R. The composition of anal basal pressure. An in vivo and in vitro study in man. International journal of colorectal disease 4: 118-22. 1989.

23. Loder P.B., Kamm M.A., Nicholls R.J., Phillips R.K. Haemorrhoids: pathology, pathophysiology and aetiology. The British journal of surgery 81: 946-54. 1994.

24. Lorenzo-Rivero S. Hemorrhoids: diagnosis and current management. Am. Surg. 75: 635-42. 2009.

25. Mentes B.B., Gorgul A., Tatlicioglu E., Ayoglu F., Unal S. Efficacy of calcium dobesilate in treating acute attacks of hemorrhoidal disease. Dis. Colon Rectum 44: 1489-95. 2001.

26. Meyer O.C. Safety and security of Daflon $500 \mathrm{mg}$ in venous insufficiency and in hemorrhoidal disease. Angiology 45: 579-84. 1994.

27. Misra M.C., Imlitemsu. Drug treatment of haemorrhoids. Drugs 65: 1481-91. 2005.

28. Misra M.C., Parshad R. Randomized clinical trial of micronized flavonoids in the early control of bleeding from acute internal haemorrhoids. Br. J. Surg. 87: 868-72. 2000.

29. Perez-Miranda M., Gomez-Cedenilla A., Leon-Colombo T., Pajares J., Mate-Jimenez J. Effect of fiber supplements on internal bleeding hemorrhoids. Hepato-gastroenterology 43: 1504-7. 1996.

30. Read M.G., Read N.W., Haynes W.G., Donnelly T.C., Johnson A.G. A prospective study of the effect of 
Man et al., Afr J Tradit Complement Altern Med. (2013) 10(2):343-351

http://dx.doi.org/10.4314/ajtcam.v10i2.21

haemorrhoidectomy on sphincter function and faecal continence. The British journal of surgery 69: 396-8. 1982.

31. Sobhani I., Walker F., Aparicio T., Abramowitz L., Henin D., Cremieux A.C., Soule J.C. Effect of anal epidermoid cancer-related viruses on the dendritic (Langerhans') cells of the human anal mucosa. Clin. Cancer Res. 8: 2862-9. 2002.

32. Sun W.M., Read N.W., Shorthouse A.J. Hypertensive anal cushions as a cause of the high anal canal pressures in patients with haemorrhoids. The British journal of surgery 77: 458-62. 1990.

33. Szlavy L., Repa I., Lengyel I., Lamboy L. Calcium dobesilate (CLS 2210) protects the myocardium in early acute myocardial infarction: a preliminary randomized, double-blind, placebo-controlled study of its effects on biochemical markers. J. Cardiovasc. Pharmacol. 15: 89-95. 1990.

34. Tchacondo T., Karou S.D., Batawila K., Agban A., Ouro-Bang'na K., Anani K.T., Gbeassor M., de Souza C. Herbal remedies and their adverse effects in Tem tribe traditional medicine in Togo. Afr. J. Tradit. Complement. Altern. Med. 8: 45-60. 2011.

35. Thomson W.H. The nature of haemorrhoids. The British journal of surgery 62: 542-52. 1975.

36. Thulesius O., Gjores J.E. Arterio-venous anastomoses in the anal region with reference to the pathogenesis and treatment of haemorrhoids. Acta chirurgica Scandinavica 139: 476-8. 1973.

37. Tong Z.Q., Yang B., Chen B.Y., Zhao M.L. A multi-center, randomized, single-blind, controlled clinical study on the efficacy of composite sophora colon-soluble capsules in treating ulcerative colitis. Chin. J. Integr. Med. 16: 486-92. 2010.

38. Webster D.J., Gough D.C., Craven J.L. The use of bulk evacuant in patients with haemorrhoids. The British journal of surgery 65: 291-2. 1978.

39. Zhao W.C., Song L.J., Deng H.Z. Protective effect of total alkaloids of Sophora alopecuroides on dextran sulfate sodium-induced chronic colitis. Chin. J. Integr. Med. 17: 616-24. 2011. 